\title{
ASSOCIAÇÃO DA IDADE E TEMPO DE INTERNAÇÃO COM TIPO DE PARTO E ABORTOS
}

\author{
ASSOCIATION OF AGE AND TIME OF INTERVENTION WITH KIND OF BIRTH \\ AND ABORTES
}

\author{
Hemilena Carmo Silva Santos', Rosilane Dias Souza ${ }^{2}$, Rodrigo Santos de Queiroz', Cleber \\ Souza de Jesus ${ }^{1}$
}

\author{
Universidade Estadual do Sudoeste da Bahia ${ }^{1}$ - UESB, IPROFIT EDUCAÇÃO CORPORATIVA ${ }^{2}$
}

\begin{abstract}
The choice of delivery type may influence cost increases, risks to maternal and child health, and should be consistent with national and international policies. The objective of the present study is to analyze the characteristics of the obstetric care of a General Hospital in the interior of Bahia and to verify the association between length of hospital stay, type of delivery and abortion. This is a retrospective and analytical study that evaluated data from 281 medical records over a period of one year. Out of the total obstetric care, 135 (48.0\%) were cesarean deliveries, 106 (37.7\%) were natural and 40 (14.3\%) were hospitalized for abortion. Women who underwent cesarean delivery $(O R=$ 2.37, 95\% Cl: 1.6-3.5) and had an abortion (OR = 2.38, 95\% Cl: 1.6-3.53) remained longer hospitalized compared with women who performed natural childbirth. Thus, a high frequency of cesarean delivery and abortions were observed in the studied sample, which contributed to an increase in the length of hospital stay. There is a clear need for more effective actions to adapt to the norms of the Prenatal and Birth Humanization Program, especially regarding the incentive to natural childbirth.
\end{abstract}

Key words: Epidemiology; Cesarean section; Normal birth; Obstetrics; Abortion

\section{Resumo}

A escolha do tipo de parto pode influenciar em aumento de custos, riscos à saúde maternoinfantil $e$ deve estar em conformidade com políticas nacionais e internacionais. $O$ objetivo do presente estudo é analisar as características dos atendimentos obstétricos de um Hospital Geral do interior da Bahia e verificar a associação entre tempo de internação, tipo de parto e aborto. Trata-se de um estudo retrospectivo e analítico que avaliou dados de 281 prontuários num período de um ano. Do total de atendimento obstétricos, 135 (48,0\%) foram partos cesáreos, 106 (37,7\%) naturais e 40 (14,3\%) internações por aborto. As mulheres que realizaram parto cesáreo (OR = 2,37; IC95\%: 1,6-3,5) e abortaram IOR = 2,38; IC95\%: 1,6-3,53) permanecem mais tempo internadas no hospital em comparação com as mulheres que realizaram parto natural. Assim, percebe-se elevada frequência de parto cesáreo e abortos na amostra estudada, o que contribuiu para um aumento no tempo de internação hospitalar. Existe clara necessidade de ações mais efetivas para adequação às normas do Programa de Humanização no Prénatal e Nascimento, principalmente com relação ao incentivo ao parto natural.

Palavras chave: Epidemiology, Cesarean section; Normal birth; Obstetrics, abortion voluntários 
Introdução

O Brasil apresenta elevada incidência de partos cesáreos, estando em desconformidade com as recomendações da Organização Mundial de Saúde $(\mathrm{OMS})^{1}$. Outro importante problema dentro desse contexto é a gravidez na adolescência, que tem sido associada a uma frequência aumentada de resultados obstétricos adversos, tais como: baixo peso ao nascer, parto prematuro, morte materna/perinatal e préeclâmpsia ${ }^{2}$.

Frente ao desafio de uma gestação e parto mais seguros, ao longo das últimas décadas vem sendo aplicadas políticas públicas voltadas para a atenção à saúde da mulher ${ }^{1,3}$. O Programa de Humanização do Pré-natal e Nascimento (PHPN) criado pelo Ministério da Saúde em junho de 2000, representa uma estratégia para facilitar o acesso das gestantes aos serviços de saúde. Ao aderirem a esse programa, os municípios recebem incentivos financeiros do Governo Federal e tem o compromisso de estruturar uma rede de atenção materno-infantil ${ }^{4}$.

Mesmo após a implantação do PHPN no Brasil, as taxas de cesárea variam bastante entre as regiões, principalmente quando se compara a assistência realizada pelo Sistema único de Saúde (SUS) com a assistência privada. A taxa de cesarianas no setor de saúde suplementar chega próximo de $80 \%$, enquanto no SUS fica próxima de $30 \%{ }^{5}$.

A Investigação da predominância dos tipos de parto e fatores associados pode contribuir para verificar se as estratégias das políticas públicas para incentivo ao parto natural e consequente melhoria da saúde materno-infantil estão sendo efetivas. Assim, o objetivo do estudo é analisar as características dos atendimentos obstétricos do Hospital Geral de Ipiaú e verificar associação entre idade e tempo de internamento com tipo de parto realizado e aborto.

\section{Metodologia}

Trata-se de estudo retrospectivo e com abordagem analítica, foi realizado através de dados secundários de internamentos para procedimentos obstétricos arquivados no Serviço de Arquivo Médico e Estatística (SAME) do Hospital Geral do interior da Bahia.

Situado na região sudoeste da Bahia, o município fica a 273,2 Km de Salvador, possui base econômica agraria e baixos indicadores de desenvolvimento socioeconômico. É um hospital público de médio, possui 10 leitos de internação obstétrica e assiste o município de Ipiaú e cidades circunvizinhas.

Foram incluídos todos os 281 casos de trabalho de parto registrados no hospital no período de 01 de janeiro a 31 de dezembro de 2012. Foram registrados, num formulário próprio, a idade (anos), a via de parto (natural e cesáreo), ocorrência de aborto e o tempo de internação.

Os dados foram coletados entre os meses de maio a junho de 2013, posteriormente tabulados e analisados através do programa estatístico SPSS for Windows 21.0. Inicialmente foi realizada uma análise descritiva através de frequências para variáveis categóricas, média, desvio padrão e valores mínimos e máximos para variáveis quantitativas.

As associações entre grupos (parto natural, parto cesáreo e aborto) com idade e tempo de internação foram testadas, inicialmente, por regressão logística multinominal bivariada (associação bruta), permanecendo para a análise multivariada apenas as variáveis que apresentaram $p \leq 0.20$. Em todas as etapas de análise foram estimadas as odds ratio com Intervalo de Confiança (IC) de 95\%. Após o ajuste, permaneceram apenas as variáveis que apresentaram $p \leq 0.05$.

O estudo foi aprovado pelo Comitê de Ética em Pesquisa (CEP) da Universidade Estadual do Sudoeste da Bahia (no de CAAE 15428313.4.0000.0055), respeitando os aspectos éticos e legais que envolvem as pesquisas com seres humanos e atende o preconizado pela resolução no 466/12 do Conselho Nacional de saúde (CNS).

\section{Resultados e Discussões}

A idade variou entre 13 e 43 anos, com média de 25 anos. 0 tempo médio de internação foi de 2,0 $\pm 2,2$ dias, com mínimo de 0 e máximo de 34 dias. Das 281 pacientes, 135 (48,0\%) realizaram parto cesáreo, $106(37,7 \%)$ realizaram partos naturais e 40 (14,3\%) foram internadas devido a abortos. Não houve registro de óbito na amostra (Tabela 1).

Nos resultados da regressão logística multinominal multivariada (Tabela 2) pode-se observar que mulheres submetidas ao parto cesáreo apresentam 2,37 vezes mais chances de permanecerem internadas por mais tempo quando comparadas com o grupo parto natural. Ainda na Tabela 2, observa-se também que as mulheres que abortaram possuem 2,38 vezes 
mais chances de permanecerem internadas por mais tempo quando comparadas com as mulheres do grupo parto natural. As mulheres do grupo aborto eram mais velhas que o grupo parto natural (Tabela 2).

Tabela 01- Média e desvio padrão da idade (anos) e tempo de internação (dias) estratificado em tipo de parto e aborto.

\begin{tabular}{llll}
\hline & Natural & Cesáreo & Aborto \\
\hline Idade & $25,2 \pm 6,6$ & $25,0 \pm 6,0$ & $28,3 \pm 7,4$ \\
\hline $\begin{array}{l}\text { Tempo de } \\
\text { internação }\end{array}$ & $1,5 \pm 1,3$ & $2,2 \pm 0,7$ & $2,4 \pm 5,2$ \\
\hline
\end{tabular}

Fonte: SAME do Hospital Geral do Interior da Bahia (Arquivos), 2012.

Tabela 2 - Resultados da análise multivariada entre tipos de parto com relação à idade e tempo de internação.

\begin{tabular}{|c|c|c|c|c|}
\hline & $\begin{array}{ll}\text { Natural } & \mathrm{x} \\
\text { Cesário }\end{array}$ & $P$ & $\begin{array}{l}\text { Natural } \mathrm{x} \\
\text { Aborto }\end{array}$ & $p$ \\
\hline & $\begin{array}{l}\mathrm{OR}_{\text {ajustado }}(\mathrm{IC} \\
95 \%)\end{array}$ & & $\begin{array}{l}\mathrm{OR}_{\text {ajustado }}(\mathrm{IC} \\
95 \%)\end{array}$ & \\
\hline Idade & $\begin{array}{l}1,00 \\
1,05)\end{array}$ & 0,71 & $\begin{array}{l}1,09 \\
1,15)\end{array}$ & $\begin{array}{l}0,00 \\
6\end{array}$ \\
\hline $\begin{array}{l}\text { Tempo } \\
\text { de } \\
\text { interna } \\
\text { ção }\end{array}$ & $\begin{array}{l}2,37 \quad(1,6- \\
3,5)\end{array}$ & $\begin{array}{l}<0,0 \\
01\end{array}$ & $\begin{array}{l}2,38 \\
3,53)\end{array}$ & $\begin{array}{l}<0,0 \\
01\end{array}$ \\
\hline
\end{tabular}

(Arquivos), 2012.

Este estudo evidenciou uma elevada frequência de partos cesáreos e abortos no Hospital Geral. As mulheres que realizaram parto cesáreo e que abortaram apresentaram duas vezes mais chances de permanecerem mais tempo internadas no hospital quando comparadas ao grupo parto natural. Observou-se ainda que as mulheres que abortaram possuíam idade mais elevada que o grupo parto natural. Não houve diferença estatisticamente significativa entre as idades das mulheres do grupo parto cesáreo e parto natural.

Os números de parto cesáreo evidenciados no presente estudo (48\%) são bem mais elevados que os $15 \%$ preconizados pela OMS. Estudos anteriores apontam que os números de partos cesáreos e abortos no Brasil são considerados alarmantes, contribuindo para o aumento de riscos e de custos relacionados à saúde maternoinfantil além de aumento de custos ${ }^{5,6,7,8}$. Dentre as justificativas para percentuais tão elevados de parto cesáreo no Brasil estão: conveniência para a mulher e para o médico, pois podem programar a data do nascimento ${ }^{9}$; menor tempo de parto e medo da dor por parte da gestante $09,10,11$.

Em outros estudos realizados no Brasil, frequências de parto cesáreo superiores a $50 \%$ também foram encontradas ${ }^{8}$. Um estudo realizado no Rio Grande do Sul demostrou que $59,24 \%$ dos tipos de parto realizado eram cesarianas. Em um estudo nacional, realizado com mulheres de diversas regiões brasileiras, observou-se um percentual de $53,4 \%$ de cesarianas $^{12}$, sendo uma realidade tanto do setor público quanto privado ${ }^{13}$.

Mesmo com os esforços do MS, que lançou uma portaria em março de 2016 com recomendações baseadas em evidências científicas sobre importantes questões relacionadas à cesariana existem dificuldades na adesão ao parto natural ${ }^{14}$. Taxas populacionais de operação cesariana superiores a $10 \%$ não contribuem para a redução da mortalidade materna, perinatal ou neonatal e estão mais associadas às questões de conveniência e ao aumento de custos em saúde. A escolha pela cesariana deve ser ponderada, evitando sua utilização de forma desnecessária e sem razões científicas $^{15,16}$.

Pacientes que realizaram parto cesáreo permanecem mais tempo internadas em comparação com as que realizaram parto natural. A recuperação do parto natural é mais rápida, possibilitando uma redução da permanência na maternidade, diminuindo o risco de infecção hospitalar, facilitando o retorno mais precoce da mãe e do recém-nascido ao convívio familiar e reduzindo custos para o sistema de saúde $^{17}$.

Outro ponto importante observado no presente estudo foi a elevada prevalência de aborto. Segundo dados do MS o esvaziamento uterino por abortamento é o segundo procedimento obstétrico mais frequente na rede pública de saúde. No país são realizadas cerca de 240 mil internações anuais no SUS para tratamento de complicações decorrentes de aborto, gerando gastos anuais da ordem de 45 milhões de reais ${ }^{18,19}$.

Em nossos dados não foi possível determinar as reais causas e os tipos de aborto, porém estima-se que ocorram mais de um milhão de abortos inseguros ao ano no Brasil, resultando em morbimortalidade materna elevada e alto custo para tratamento das complicações. No período de 1996 a 2011 foram registradas um total de 1.284 mortes devido a abortos, sendo que $8,9 \%$ (115) dessas mortes ocorreram na Bahia $^{13,20}$. 
Um tipo de aborto comum, principalmente em regiões com menores indicadores socioeconômicos, é o aborto criminal, que é um aborto provocado em desacordo com as condições do Código Penal Brasileiro. São exceções ao aborto criminal: casos de gestações provenientes de estupro; situações quando há risco de morte para as gestantes; e casos de anomalias fetais graves incompatíveis com a vida extra-uterina. Sob essas circunstâncias, a mulher tem o direito de interromper a gestação de maneira segura, com assistência à saúde integral, ética e humanizada ${ }^{18,21,22}$.

Foi observado também que as mulheres que abortaram possuíam idade mais elevada que o grupo parto natural. Esse crescimento da proporção de abortos com a idade é apontado como um fenômeno comum na vida reprodutiva das mulheres. Estima-se que mais de um quinto das mulheres no Brasil urbano já abortaram, sendo que prevalências mais elevadas podem ser encontradas quando se estuda populações de baixa escolaridade. O abortamento espontâneo, assim como o provocado, ocorre em maior frequência nas mulheres não brancas, com mais de um filho nascido vivo e união estável. Assim, é possível considerar que, apesar da existência de políticas públicas direcionadas à atual realidade da mulher, os serviços de saúde não têm logrado alcançar êxito em ações de saúde para modificar esse quadro crítico no campo da saúde pública ${ }^{23}$.

O estudo apresenta limitações inerentes ao próprio desenho (retrospectivo), que não permitiu a determinação das reais causas que levaram ao aborto e a escolha do tipo de parto. Esses fatores poderiam ajudar nas análises e favorecer um panorama mais claro no reconhecimento de partos cesáreos desnecessários e do aborto criminal. A falta de maiores informações socioeconômicas também não possibilitou uma análise mais robusta dos fatores associados, limitando-nos ao tempo de internação e idade.

\section{Conclusão}

Neste estudo observou-se alta prevalência de aborto e de cesarianas num hospital público do interior da Bahia. Observou-se que as mulheres submetidas ao parto cesáreo e as que abortaram permaneceram mais tempo internadas no hospital. Esse cenário aponta para desconformidade com relação a orientações da Organização Mundial de Saúde e do próprio Ministério da Saúde, o que pode impactar em aumento de custos e maiores riscos à saúde materno-infantil. Esses dados demonstram a necessidade do fortalecimento de políticas públicas para incentivo ao parto natural e classificação das gestantes quanto risco obstétrico.

\section{Referências}

1. CRUZ, E.G. Perfil de Atendimento de uma Maternidade Estadual de Referencia Secundária de Alto Risco em Caruaru, Pernambuco. Recife 2010.

2. CECCONELLO, F; FERRAZ, L. O perfil sócio-demográfico e patológico de gestantes e puérperas admitidas na unidade de terapia intensiva de um hospital do oeste catarinense. Ágora: R. Divulg. Cient., Mafra, v. 17, n. 1, 2010.

3. BRASIL. Ministério da Saúde. Secretaria de Políticas de Saúde. Área Técnica de Saúde da Mulher. Parto, Aborto e Puerpério: assistência humanizada a mulher. Brasília, DF; 2003.

4. BRASIL. Ministério da Saúde. Secretaria de Políticas de Saúde. Programa de humanização no pré-natal e nascimento. Humanização no Parto. Brasília - DF; 2000.

5. AMORIM, M.M.R; SOUZA, A.S; PORTO, A.M.F. Indicações de cesariana baseadas em evidências: parte I. Rev. Feminina, Recife 2010.

6. RIQUINHO, D; CORREIA, S.G. Mortalidade materna: perfil sócio-demográfico e causal. Rev Bras Enferm, 2006 maio-jun; 59(3): 303-7

7. World Health Organization. Classificação Estatística Internacional de Doenças e Problemas Relacionados à Saúde; 10ạ Revisão. CBCD, São Paulo; 1995.

8. BRASIL. Ministério da Saúde. Atenção à saúde do recém-nascido: guia para os profissionais de saúde. Brasília - DF; 2011

9. RENNER, F.W; et al. Perfil epidemiológico das puérperas e dos recémnascidos atendidos na maternidade de um hospital de referência do interior do Rio Grande do Sul no primeiro semestre de 2014. Bol Cient Pediatr, 2015. 04(2):27-32.

10. PÀDUA, K.S; et al. Fatores associados à cesariana em hospitais brasileiros. Rev. Saúde Pública 2010; 44(1):70-9.

11. FREITAS, PF et al. Fatores médicos e nãomédicos associados às cesarianas em um hospital universitário no Sul do Brasil. Caderno de Saúde Pública, Rio de Janeiro, 24(5):1051-1061, 2008. 
12. D'ORSI. E; et al. Desigualdades sociais e satisfação das mulheres com o atendimento no parto no Brasil: estudo nacional de base hospitalar. Caderno Saúde Pública, Rio de Janeiro, 2014. Vol 30.

13. DATASUS, 2012. Disponível em <http://tabnet.datasus.gov.br/cgi/tabcgi.exe?cne s/cnv/leiintbr.def> Acessado em 22/08/2016

14. BRASIL. Ministério da saúde, secretaria de atenção à saúde portaria 306, de 28 de março de 2016. Aprova as Diretrizes de Atenção à Gestante: a operação cesariana. Brasília-DF, 2016 15. FAÚNDES, A; CECATTI, J.G. A operação Cesárea no Brasil. Incidência, tendências, causas, conseqüências e propostas de ação. Cad. Saúde Pública vol.7 no.2 Rio de Janeiro Apr./June 1991.

16. OSAVA, R.H; et al. Caracterização das cesarianas em centro de parto normal. Revista de Saúde Pública, 2011; 45(6): 1036-43.

17. BRASIL. Ministério da saúde, agencia nacional de Saúde Suplementar -ANS. Media de permanência maternidade.V.1Brasília-DF, 2013.

18. BRASIL. Ministério da Saúde. Secretaria de Atenção à Saúde. Prevenção e tratamento dos agravos resultantes da violência sexual contra mulheres e adolescentes. 2. ed. Atualizada e ampliada. Brasília. 2005.

19. ANJOS, K. F.; et al. Aborto e saúde pública no Brasil: reflexões sob a perspectiva dos direitos humanos. Saúde em Debate. Rio de Janeiro, v. 37, n. 98, p. 504-515, jul/set 2013

20. BRASIL. Ministério da Saúde. Secretaria de Atenção à Saúde. Abortamento previsto em lei em situações de violência sexual - Brasília: Ministério da Saúde, 2008.

21. FRIGÉRIO M.V; et al. Aspectos bioéticos e jurídicos do abortamento seletivo no Brasil. In: Aborto Legal - Implicações éticas e religiosas. São Paulo, Católicas pelo Direito de Decidir, 2002.

22. RAMOS, K. S. Mulheres hospitalizadas por abortamento em uma maternidade-escola na cidade do recife. Rev Esc Enferm USP 2010; 44(3):605-10

23. SANTOS, V.C; et al. Criminalização do aborto no Brasil e implicações à saúde pública. Revista Bioética, v. 21, n. 3, p. 494-508, 2013.

\section{Endereço para Correspondência}

Universidade Estadual do Sudoeste da Bahia UESB

Rua Av. José Moreira Sobrinho - Jequiezinho, Jequié - BA

CEP.: $45205-490$

e-mail hemilla3@yahoo.com.br

Recebido em 26/04/2017

Aprovado em 09/11/2017

Publicado em 01/12/2017 